\title{
Overexpression of CD44 Standard Isoform Upregulates HIF-1 $\alpha$ Signaling in Hypoxic Breast Cancer Cells
}

\author{
Dayoung Ryu' ${ }^{1}$ In-geun Ryoo ${ }^{2}$ and Mi-Kyoung Kwak ${ }^{1,2,3, *}$ \\ ${ }^{1}$ Department of Pharmacy, Graduate School of The Catholic University of Korea, Bucheon 14662, \\ ${ }^{2}$ Integrated Research Institute for Pharmaceutical Sciences, The Catholic University of Korea, Bucheon 14662, \\ ${ }^{3}$ College of Pharmacy, The Catholic University of Korea, Bucheon 14662, Republic of Korea
}

\begin{abstract}
Cluster of differentiation 44 (CD44), a cell surface receptor for hyaluronic acid (HA), is involved in aggressive cancer phenotypes. Herein, we investigated the role of the CD44 standard isoform (CD44s) in hypoxia-inducible factor-1 $\alpha$ (HIF-1 $\alpha)$ regulation using MCF7 overexpressing CD44s (pCD44s-MCF7). When pCD44s-MCF7 was incubated under hypoxia, levels of HIF-1 $\alpha$, vascular endothelial growth factor, and the HIF-1 $\alpha$ response element-derived luciferase activity were significantly increased compared to those in the control MCF7. Incubation of pCD44s-MCF7 cells with HA further increased HIF-1 $\alpha$ accumulation, and the silencing of CD44s attenuated HIF-1 $\alpha$ elevation, which verifies the role of CD44s in HIF-1 $\alpha$ regulation. In addition, the levels of phosphorylated extracellular signal-regulated kinase (ERK) was higher in hypoxic pCD44s-MCF7 cells, and HIF-1 $\alpha$ accumulation was diminished by the pharmacological inhibitors of ERK. CD44s-mediated HIF-1 $\alpha$ augmentation resulted in two functional outcomes. First, pCD44s-MCF7 cells showed facilitated cell motility under hypoxia via the upregulation of proteins associated with epithelialmesenchymal transition, such as SNAIL1 and ZEB1. Second, pCD44s-MCF7 cells exhibited higher levels of glycolytic proteins, such as glucose transporter-1, and produced higher levels of lactate under hypoxa. As a consequence of the enhanced glycolytic adaptation to hypoxia, pCD44s-MCF7 cells exhibited a higher rate of cell survival under hypoxia than that of the control MCF7, and glucose deprivation abolished these differential responses of the two cell lines. Taken together, these results suggest that CD44s activates hypoxia-inducible HIF-1 $\alpha$ signaling via ERK pathway, and the CD44s-ERK-HIF-1 $\alpha$ pathway is involved in facilitated cancer cell viability and motility under hypoxic conditions.
\end{abstract}

Key Words: CD44s, HIF-1 $\alpha$, ERK, Hypoxia, Glycolysis, Cell motility

\section{INTRODUCTION}

The glycoprotein cluster of differentiation 44 (CD44) is a cell surface receptor for several extracellular matrix (ECM) proteins, such as hyaluronic acid, and is highly expressed in various cancers, participating in cancer cell proliferation and metastasis (Naor et al., 2002; Zöller, 2011; Yan et al., 2015). Although human CD44 gene is a single and conserved gene located on chromosome 11, structural and functional diversities occur from alternative splicing and post-translational glycosylation, which appears to endow differential biological roles to each CD44 isoform (Naor et al., 2002; Yan et al., 2015). The CD44 standard isoform (CD44s) consists of the conserved exons of $C D 44$ gene and is expressed in a wide array of animal cells, participating in cell-cell interactions and cell-matrix in- teractions. In cancer cells, high CD44s level has been associated with cancer stem cell (CSC)-like properties such as facilitated cell motility, invasion, and migration (Tsukita et al., 1994; Ohashi et al., 2007; Karousou et al., 2017). Prostate cancer cells with high CD44 expression exhibited elevated levels of $\beta$-catenin and octamer-binding protein 3/4 (OCT3/4), which are markers of CSCs, and these cells were highly proliferative, tumorigenic, and metastatic compared to the prostate cancer cells with low CD44 expression (Patrawala et al., 2006). Gastric cancer cells with CD44 overexpression retained high activity of hedgehog signaling and were relatively resistant to chemotherapeutic drugs such as cisplatin and 5-fluorouracil (Yoon et al., 2014). In addition, CD44 has been known to induce the epithelial-to-mesenchymal transition (EMT) of breast, liver, gastric, and pancreatic cancer cells (Cho et al., 2012; Zhang

\section{Open Access https://doi.org/10.4062/biomolther.2018.116}

This is an Open Access article distributed under the terms of the Creative Commons Attribution Non-Commercial License (http://creativecommons.org/licenses/by-nc/4.0/) which permits unrestricted non-commercial use, distribution, and reproduction in any medium, provided the original work is properly cited.
Received Jun 26, 2018 Revised Jul 6, 2018 Accepted Jul 16, 2018 Published Online Sep 1, 2018

\section{*Corresponding Author}

E-mail:mkwak@catholic.ac.kr

Tel: +82-2-2164-6532, Fax: +82-2-2164-4059 
et al., 2012; Shang et al., 2015). Overexpression of CD44s induced tumor invasion of hepatocellular carcinoma cells along with the elevation of mesenchymal marker vimentin (Mima et al., 2012). CD44s has been identified as a clinical prognostic factor. Colorectal cancer patients with high CD44s expression showed reduced overall survival rates (Huh et al., 2009).

Intra-tumoral hypoxic environment promotes aggressive progression of cancer cells by enhancing tumor angiogenesis, cell motility, and survival (Semenza, 2004). Hypoxia-inducible factor-1 (HIF-1) is a major transcription factor that senses low environmental $\mathrm{O}_{2}$ and induces adaptive cellular programing to hypoxic condition. HIF-1 is a heterodimeric complex composed of $\mathrm{HIF}-1 \alpha$, which is regulated by $\mathrm{O}_{2}$ concentration, and HIF-1 $\beta$ (aryl hydrocarbon receptor nuclear translocator, ARNT), which is constitutively expressed (Guillemin and Krasnow, 1997; Semenza, 1999). Under normal $\mathrm{O}_{2}$ levels, HIF-1 $\alpha$ is continuously degraded by the prolyl hydroxylase domain proteins (PHD)-mediated hydroxylation and subsequent degradation via ubiquitinylation by the Von Hippel-Lindau ( $\mathrm{pVHL}$ ) tumor suppressor protein. Under hypoxic conditions, PDHmediated HIF-1 $\alpha$ hydroxylation is blocked due to lack of $\mathrm{O}_{2}$, which is a substrate of PDH, and therefore, HIF- $1 \alpha$ accumulates and transactivates HIF-1 $\alpha$ response element (HRE)containing genes (Weidemann and Johnson, 2008). HIF-1 $\alpha$ target genes include angiogenesis factors such as vascular endothelial growth factor (VEGF), glucose metabolism factors, such as pyruvate dehydrogenase kinase 1 (PDK1) and glucose transporter 1 (GLUT1), and EMT factors, such as SNAIL1 and ZEB1 (Guillemin and Krasnow, 1997; Semenza, 1999; Weidemann and Johnson, 2008). In particular, the HIF$1 \alpha$-mediated hypoxia-inducible expression of GLUT1 and glycolytic enzymes endow cancer cells with higher survival capacity in $\mathrm{O}_{2}$-limited condition by facilitating glucose metabolism for ATP production (Semenza, 2013).

Several reports have demonstrated the link between hypoxia-inducible signaling and CD44. Hypoxic stimulation of ovarian cancer cells increased CD44 expression along with expression of markers responsible for imparting stemness to the cells (Liang et al., 2012). Repeated exposure of metastatic breast cancer cells to hypoxic conditions resulted in the enrichment of cancer cells with high CD44 expression (Louie et al., 2010). In triple negative breast cancer cells, hypoxia upregulated the expression of CD44 variant isoform as has been verified from human tumor samples (Krishnamachary et al., 2012). These results suggest that CD44 is upregulated during hypoxia via HIF-1 $\alpha$, which contributes to HIF-1 $\alpha$-mediated cancer progression. However, there has been no report showing the effect of CD44s on HIF-1 $\alpha$ signaling and hypoxic response of cancer cells. In this study, we have established stable breast cancer MCF7 overexpression cell line for CD44s, and examined its hypoxic response and $\mathrm{HIF}-1 \alpha$ regulation.

\section{MATERIALS AND METHODS}

\section{Materials}

Hyaluronic acid (HA) sodium salt, Z-Leu-Leu-Leu-al (MG132), and U0126 were obtained from Sigma-Aldrich Co (St. Louis, MO, USA). PD98059 was purchased from Calbiochem (Billerica, MA, USA). The luciferase reporter plasmid containing 5 copies of the human VEGF HRE was a gift from Dr. You Mie Lee (Kyungpook National University, Daegu, Ko- rea). The HIF- $1 \alpha$ antibody was purchased from BD Biosciences (Palo Alto, CA, USA). VEGF, aryl hydrocarbon receptor nuclear translocator (ARNT), and $\beta$-tubulin antibodies were purchased from Santa Cruz Biotechnology (Santa Cruz, CA, USA). Antibodies for Twist-related protein 1 (TWIST) and zinc finger E-box binding homeobox 1 (ZEB1) were obtained from GeneTex (Irvine, CA, USA). Antibodies for hexokinase (HK2), pyruvate dehydrogenase kinase 1 (PDK1), lactate dehydrogenase A (LDHA), glucose transporter 1 (GLUT1), SNAIL1, extracellular-signal-regulated kinase (ERK), phosphorylated ERK (p-ERK), AKT, p-AKT, p38, p-p38, c-Jun N-terminal kinase (JNK), and p-JNK were purchased from Cell Signaling Technology (Danvers, MA, USA).

\section{Cell culture}

The human breast carcinoma cell line MCF7 was obtained from the American Type Culture Collection (Rockville, MD, USA). MCF7 cells were grown in Dulbecco's modified Eagle's medium (DMEM) supplemented with $10 \%$ fetal bovine serum (FBS; Hyclone, Logan, UT, USA) and penicillin/streptomycin (WelGene Inc., Daegu, Korea). The cells were maintained at $37^{\circ} \mathrm{C}$ in an atmosphere of $5 \% \mathrm{CO}_{2}$. The stable cell lines with the control plasmid (pBabe) or the CD44 expression plasmid ( $p C D 44 s-B a b e)$ were established following the retrovirus packaging mixture and puromycin selection (Ryoo et al., 2018).

\section{Western blot analysis}

After incubation, cells were lysed with radioimmunoprecipitation assay (RIPA) buffer containing protease/phosphatase inhibitors. The protein concentration was determined using a bicinchoninic acid assay (BCA) kit (Thermo Scientific, Middletown, VA, USA). The obtained protein samples were separated by $6 \%$ or $10 \%$ SDS-polyacrylamide gels, and then transferred to nitrocellulose membranes (Whatman $\mathrm{GmbH}$, Dassel, Germany). The membranes were then incubated with the primary antibody overnight following incubation with $3 \%$ bovine serum albumin (BSA) for $1 \mathrm{~h}$. Then, the membranes were incubated with the corresponding secondary antibody for $1 \mathrm{~h}$. Chemiluminescent images were detected using a LAS4000 mini imager (GE Healthcare Life Sciences, Piscataway, NJ, USA) as described previously (Park et al., 2017).

\section{HRE luciferase reporter assay}

Cells were seeded on a 24 -well plate at a density of $2 \times 10^{4}$ cells/well and incubated overnight. The cells were then transfected using a mixture of $0.5 \mu \mathrm{g}$ HRE-luciferase reporter plasmid, $0.05 \mu \mathrm{g}$ pRLtk control plasmid (Promega, Madison, WI, USA), and the transfection reagent (WelGene Inc.). After 18 $\mathrm{h}$, the transfection mixture was removed, and the cells were then recovered in a complete medium for $24 \mathrm{~h}$. Renilla and Firefly luciferase activities were monitored in cell lysates with the Dual Luciferase Assay System (Promega) as reported previously (Kim et al., 2011).

\section{siRNA transfection}

Predesigned CD44s siRNA (siCD44s; 5'-CACUGUUUUCAACCUCGAA-3' and 5'-UUCGAGGUUGAAAACAGUG-3') and scrambled control siRNA ( $\mathrm{SiCN}$ ) were obtained from Bioneer Corporation (Daejeon, Korea). Cells seeded in a 6-well plate were transfected with either siCN or siCD44s using a Lipofectamine 2000 reagent (Life Technologies, Carlsbad, CA, 
A
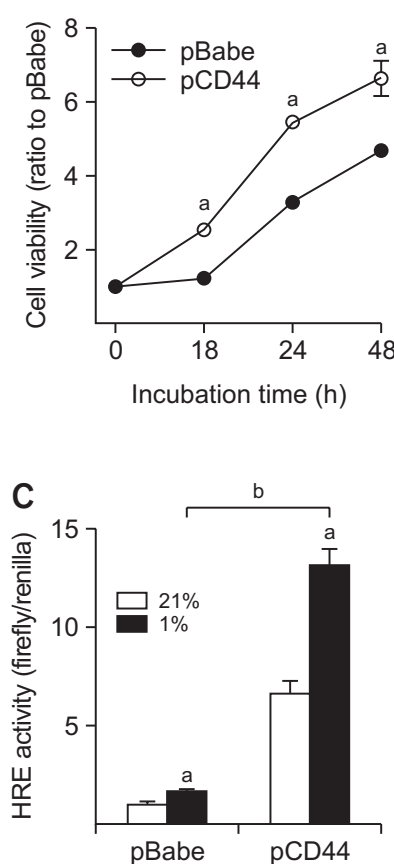

B

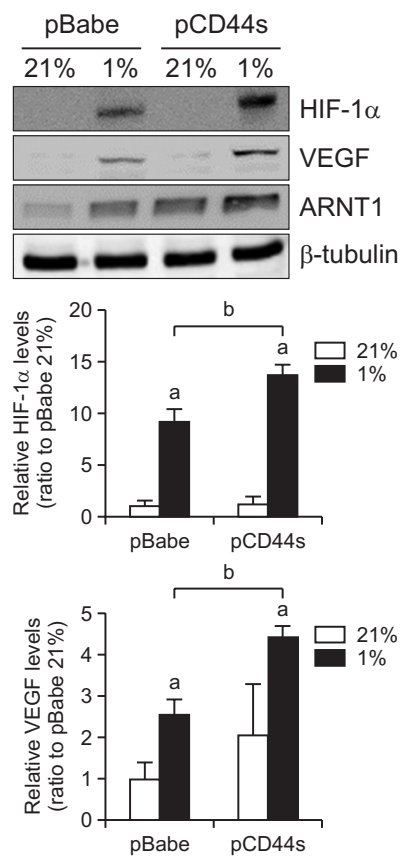

Fig. 1. Hypoxia-induced HIF-1 $\alpha$ accumulation is enhanced in CD44s-overexpressing MCF7. (A) Cell numbers were counted after 18,24 , or $48 \mathrm{~h}$ of the plating of pBabe-MCF7 control and pCD44s-MCF7 cells. Values are represented as mean \pm standard error (SE). (B) The pBabe-MCF7 control and pCD44s-MCF7 cells were incubated in $21 \% \mathrm{O}_{2}$ (normoxia) or $1 \% \mathrm{O}_{2}$ (hypoxia) condition for $24 \mathrm{~h}$, and protein levels of HIF-1 $\alpha$, VEGF, and ARNT1 were determined using western blot. The quantified relative levels are represented as mean \pm SE from three independent experiments. (C) HRE-derived luciferase activity was assessed in normoxic and hypoxic cell lines. Values are represented as mean \pm SE from three independent experiments. ${ }^{a} p<0.05$ compared to each normoxic cell line. ${ }^{b} p<0.05$ compared to the hypoxic pBabe-MCF7 control.

USA). The next day, the transfection complex-containing medium was removed and the cells were recovered after incubation in complete medium overnight.

\section{Wound healing assay}

To determine cell motility, the pBabe-MCF7 and pCD44sMCF7 cells were seeded in 6-well plates at a density of $8 \times 10^{5}$ cells/well. When the cells achieved $90-100 \%$ confluency, a straight scratch was made on the surface using a pipette tip. The cells were grown for 24 or $48 \mathrm{~h}$, and the migration of cells into the wounded area was photographed using a JULI ${ }^{\mathrm{TM}}$ Smart fluorescent cell analyzer (Digital Bio source, Seoul, Korea). The wound closure rate was determined using the initial and final wound widths, and the wound closure percentage was calculated by dividing the change in wound width by the initial wound width (Kang et al., 2017).

\section{Lactate measurement}

The extracellular lactate level was quantified with a Lactate Colorimetric/Fluorometric Assay Kit (BioVision, CA, USA). The complete medium from hypoxic pBabe- and pCD44s-MCF7 was deproteinized using a $10 \mathrm{kDa}$ molecular weight spin filter (BioVision) and the samples were added in a 96-well plate

A
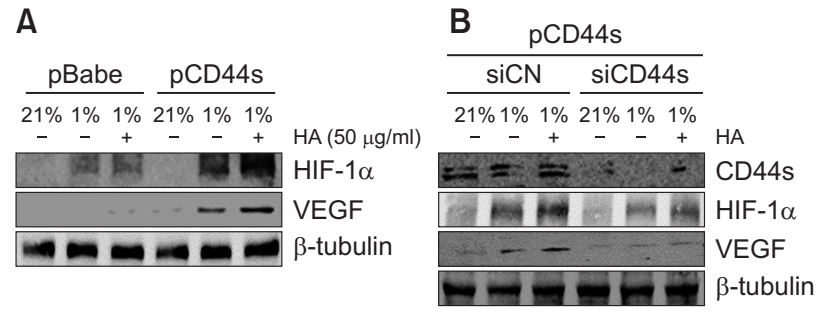

Fig. 2. CD44s mediates the enhanced HIF-1 $\alpha$ accumulation in CD44s-overexpressing MCF7 cells. (A) Protein levels of HIF- $1 \alpha$ and VEGF were determined in normoxic and hypoxic cells after incubation with HA (50 $\mu \mathrm{g} / \mathrm{ml})$ for $24 \mathrm{~h}$. (B) The pCD44s-MCF7 cells were transfected with CD44-specific siRNA (siCD44) or nonspecific control RNA (siCN), and protein levels of CD44s, HIF-1 $\alpha$, and VEGF were assessed after a $24 \mathrm{~h}$ incubation under normoxic or hypoxic condition.

(50 $\mu \mathrm{g} /$ well). Lactate enzyme mix was added into each well, and incubated for $30 \mathrm{~min}$ at room temperature in dark. Then the absorbance at $570 \mathrm{~nm}$ was quantified using a SPECTROstar $^{\circledR}$ nano plate reader (BMG LABTECH GmbH, Ortenberg Germany).

\section{Cell count}

The pBabe- and pCD44s-MCF7 were incubated in hypoxic conditions for $24 \mathrm{~h}$ and viable cells were counted using a TC10 Automated Cell Counter (Bio-Rad Laboratories, Inc., Hercules, CA, USA) after the addition of the trypan blue dye.

\section{Statistical analysis}

Statistical significance was analyzed with a one-way analysis of variance (ANOVA) followed by Student Newman-Keuls test for multiple comparisons. These analyses were done using GraphPad Prism software (GraphPad Prism, La Jolla, CA, USA).

\section{RESULTS}

\section{HIF- $1 \alpha$ accumulation is enhanced in MCF7 cells showing CD44s overexpression}

We established MCF7 stable cell lines by transfection with either a plasmid expressing the CD44s (pCD44s-MCF7) or a blank plasmid ( $\mathrm{pBabe-MCF7)} \mathrm{as} \mathrm{described} \mathrm{in} \mathrm{previous} \mathrm{inves-}$ tigation (Ryoo et al., 2018). The pCD44s-MCF7 cells showed a higher cell proliferation rate compared to the control cell line (Fig. 1A). As we observed that MCF7 cells produce low levels of HA (unpublished data), promoting effect of CD44 on cell proliferation can be a stimulating effect of HA on CD44 (Karousou et al., 2017). When these cells were incubated under hypoxic conditions $\left(1 \% \mathrm{O}_{2}\right)$ for $24 \mathrm{~h}$, inducible HIF- $1 \alpha$ level was relatively higher in CD44s compared to that in the control cells (Fig. 1B). With a similar pattern, hypoxia-inducible level of VEGF was significantly higher in pCD44s-overexpressing MCF7 cells. The level of ARNT, a transcriptional coactivator of HIF- $1 \alpha$, was also elevated during hypoxia, and the elevation fold was higher in pCD44s-MCF7 cells compared to that in pBabe-MCF7 cells. In line with these results, hypoxia-inducible activation of the hypoxia-response element (HRE)-derived luciferase was significantly high in pCD44s-MCF7 cells compared to the pBabe-MCF7 cells (Fig. 1C). These results 
suggested that $\mathrm{HIF}-1 \alpha$ accumulation is facilitated by pCD44s overexpression in breast cancer cells.

\section{CD44s mediates the activation of HIF-1 $\alpha$ upon hypoxia}

In order to confirm the effects of CD44s on HIF-1 $\alpha$ activation, HA, a major ligand of CD44, was incubated and HIF-1 $\alpha$ response was examined. When pCD44s-MCF7 cells were incubated under hypoxic conditions in the presence of HA $(50 \mu \mathrm{g} / \mathrm{ml})$, hypoxia-inducible HIF- $1 \alpha$ and VEGF levels were significantly higher in them compared to those in the hypoxic pCD44s-MCF7 in the absence of HA (Fig. 2A). On the contrary, when CD44s expression was silenced by CD44s-specific siRNA transfection, hypoxia-inducible HIF-1 $\alpha$ and VEGF elevations were suppressed in pCD44s-MCF7 cells (Fig. 2B). Augmentation effect of HA was also repressed following $C D 44 s$ silencing. These results indicate the direct involvement of CD44s in HIF- $1 \alpha$ activation.

\section{CD44s activates HIF-1 $\alpha$ signaling via ERK activation}

MAPK pathway is one of the cellular signaling pathways activated by CD44s, and this pathway has also been associated with HIF-1 $\alpha$ activation under hypoxic condition (Kietzmann et al., 2016; Chen et al., 2018). Based on that, we hypothesized that enhanced MAPK signaling might affect differential HIF$1 \alpha$ expression in CD44s overexpression MCF7. In order to test our hypothesis, cells were incubated under hypoxic conditions for $24 \mathrm{~h}$ and levels of MAPK signaling components, such as ERK, JNK, and p38 was monitored. Among them, the levels of phosphorylated ERK ( $p$-ERK) were elevated in hypoxic pCD44s-MCF7 cells, whereas no increase in its levels was observed in pBabe control cells (Fig. 3A). JNK signaling was activated by hypoxia in both group of cells with a similar pattern, and p-p38 level did not show significant changes. In addition, HA treatment in pCD44s-MCF7 cells elevated p-ERK levels in a concentration-dependent manner (Fig. 3B). These results indicated the potential involvement of ERK activation in elevation of HIF-1 $\alpha$ levels in CD44s-overexpressing MCF7 cells. Further evidence was obtained from chemical inhibitor experiment. When pCD44s-MCF7 cells were incubated with chemical ERK inhibitors such as U0126 and PD98059, hypoxia-inducible elevations in HIF- $1 \alpha$ and VEGF were repressed (Fig. 3C, 3D). In fact, the level of hypoxia-inducible HIF-1 $\alpha$ in pCD44s cells was reduced to the level of HIF-1 $\alpha$ in hypoxic control cells after U0126 treatment (Fig. 3C), which suggested that ERK signaling activation led to higher HIF-1 $\alpha$ accumulation in CD44s-overexpressing cells.

\section{CD44s leads to enhanced cancer cell motility under hypoxic conditions}

Since HIF-1 $\alpha$ expression induces cancer cell motility through the upregulation of EMT genes (Myszczyszyn et al., 2015), higher levels of HIF-1 $\alpha$ in pCD44s-MCF7 cells can be associated with differential cell motility. When wound healing assay was performed under a $24 \mathrm{~h}$ - or $48 \mathrm{~h}$-hypoxic condition, cell motility was significantly enhanced (Fig. 4). Notably, cell motility was greater in pCD44s-MCF7 than that in the control cells. Consistent with this, hypoxia-inducible levels of EMT proteins such as ZEB1, SNAIL, and TWIST were relatively higher in pCD44-MCF7 (Fig. 4B). The inhibition of ERK signaling using U0126 blocked the expression of EMT proteins in pCD44s-MCF7 (Fig. 4C), which verifies the involvement of ERK-HIF-1 $\alpha$ signaling in the motility of hypoxic cancer cells.
A

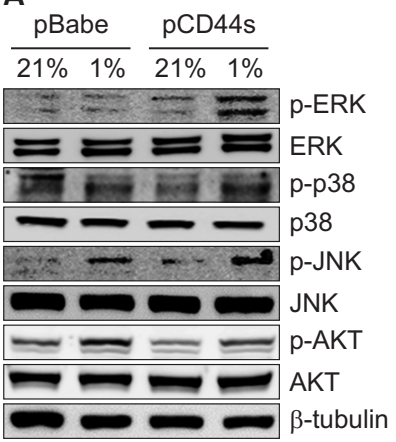

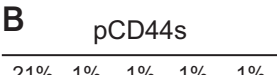
$\begin{array}{cccccc}21 \% & 1 \% & 1 \% & 1 \% & 1 \% & \\ 0 & 0 & 25 & 50 & 100 & \mathrm{HA}(\mu \mathrm{g} / \mathrm{ml})\end{array}$

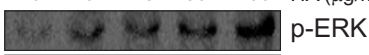

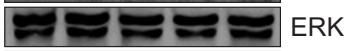

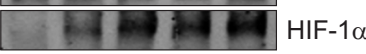
$x+m+\infty)$ VEGF $\sim \sim \beta$-tubulin

C

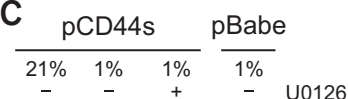
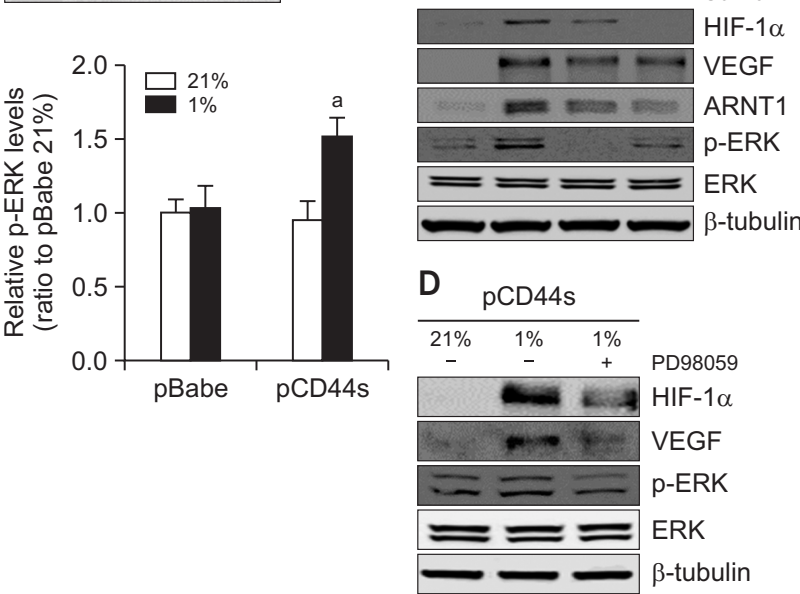

Fig. 3. ERK signaling is involved in the CD44s-mediated HIF-1 $\alpha$ accumulation. (A) Protein levels of total/phosphorylated ERK ( $p$ ERK), total/phosphorylated p38 (p-p38), total/phosphorylated JNK ( $p-J N K)$, and total/phosphorylated AKT ( $p-A K T)$ were determined in pBabe-MCF7 and pCD44s-MCF7 cells after incubation in normoxic or hypoxic condition. Quantitative protein levels are represented as mean \pm SE from three experiments. ${ }^{a} p<0.05$ compared to each normoxic cell line. (B) Levels of $p$-ERK, HIF-1 $\alpha$, and VEGF were assessed in hypoxic pCD44s-MCF7 cells after incubation with HA $(25,50$, and $100 \mu \mathrm{g} / \mathrm{mL})$. (C) Levels of HIF-1 $\alpha$, VEGF, ARNT1, and $p$-ERK were monitored in hypoxic pCD44s-MCF7 cells after incubation with a chemical inhibitor of ERK (U0126, $30 \mu \mathrm{M})$. (D) Hypoxic pCD44s-MCF7 cells were incubated with another inhibitor of ERK (PD98059, $30 \mu \mathrm{M})$, and protein levels of HIF-1 $\alpha$, VEGF, and $\mathrm{p}$-ERK were determined.

These results confirm that differential HIF- $1 \alpha$ accumulation in CD44s-overexpressing cancer cells led to enhanced cell motility via the upregulation of EMT-related factors when compared to the control cells.

\section{CD44s overexpression promotes glycolysis and cell survival under hypoxic conditions}

HIF-1 $\alpha$ promotes glycolysis by promoting transcription of the related metabolic enzymes (Semenza, 2013). Differential HIF-1 $\alpha$ accumulation in pCD44s-overexpressing cells can lead to enhanced glycolysis and cell survival. To explore this possibility, first, the cell number was assessed after their incubation under hypoxic conditions for $24 \mathrm{~h}$. Viable cell number was reduced after the hypoxic incubation in both cell lines, and it was notable that the decrease in cell number in pCD44s-MCF7 was significantly less compared to that in pBabe control (Fig. 5A). The enhanced viability of pCD44s- 
A
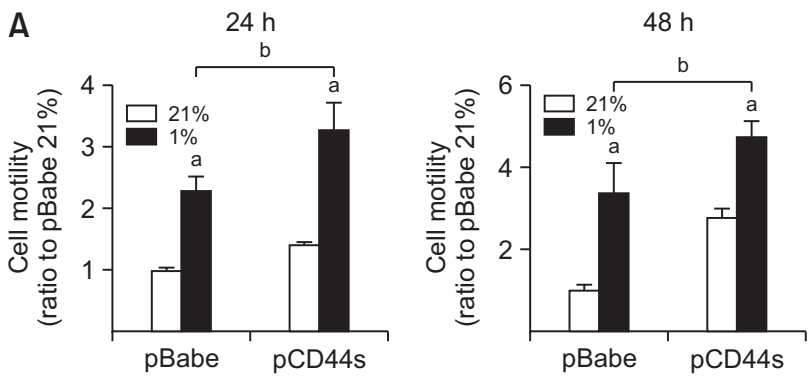

B

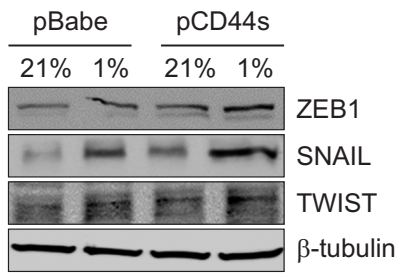

A

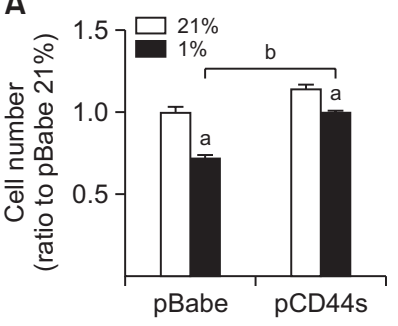

C

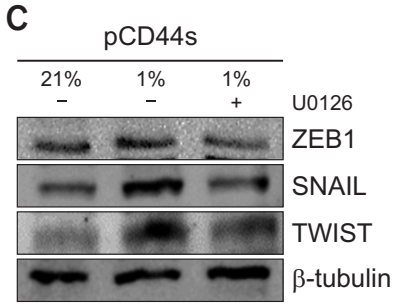

Fig. 4. CD44s leads to enhanced cancer cell motility under hypoxia. (A) Cell motility was assessed using the wound healing assay. The pBabe- and pCD44s-MCF7 cells were incubated in normoxic or hypoxic condition for $24 \mathrm{~h}$ or $48 \mathrm{~h}$, and the gap distance was measured. The wound closure percentage was calculated by dividing the change in wound width by the initial wound width. Values are represented as mean \pm SE from three independent experiments. ${ }^{a} p<0.05$ compared to each normoxic cell line. ${ }^{b} p<0.05$ compared to the hypoxic pBabe-MCF7 cell line. (B) Protein levels of ZEB1, SNAIL, and TWIST were monitored in normoxic and hypoxic cells using western blotting. (C) ZEB1, SNAIL, and TWIST protein levels were determined in U0126-treated hypoxic pCD44s-MCF7 cells.

MCF7 cells under hypoxia could be explained by the increase in levels of glycolysis-associated enzymes. Hypoxia-inducible levels of GLUT1, HK2, and PDK1 were higher in pCD44sMCF7 cells compared to those in the control cells (Fig. 5B). HA treatment further elevated GLUT1, HK2, and PDK1 levels in pCD44s-MCF7, which confirmed the role of HA-CD44s in the upregulation of glycolysis enzymes (Fig. 5C). In addition, we observed that the hypoxia-induced increase in lactate levels was significantly high in pCD44s-MCF7 cells compared to that in the control cells (Fig. 5D). These results indicate that CD44s overexpression promoted glycolysis in cancer cells, which is a beneficial metabolic change for cell survival and growth under hypoxic conditions. Indeed, glucose-dependent cell survival and growth in pCD44s-MCF7 could be confirmed by the deprivation of glucose in culture medium. The viability of pCD44s-MCF7 cells under hypoxic condition was largely reduced in glucose-free medium, whereas effect of glucose deprivation was minimal in the pBabe control cells (Fig. 5E).

\section{DISCUSSION}

CD44 plays a critical role in communicating with surrounding tumor microenvironment by functioning as a receptor for hyaluronic acid and other ECM proteins, and transduces environmental changes into intracellular signaling networks for facilitated cell growth, tumor invasion, and metastasis (Bourguignon et al., 2008; Yan et al., 2015; Chen et al., 2018). The binding of HA to CD44 induced metastasis of melanoma cells,

B
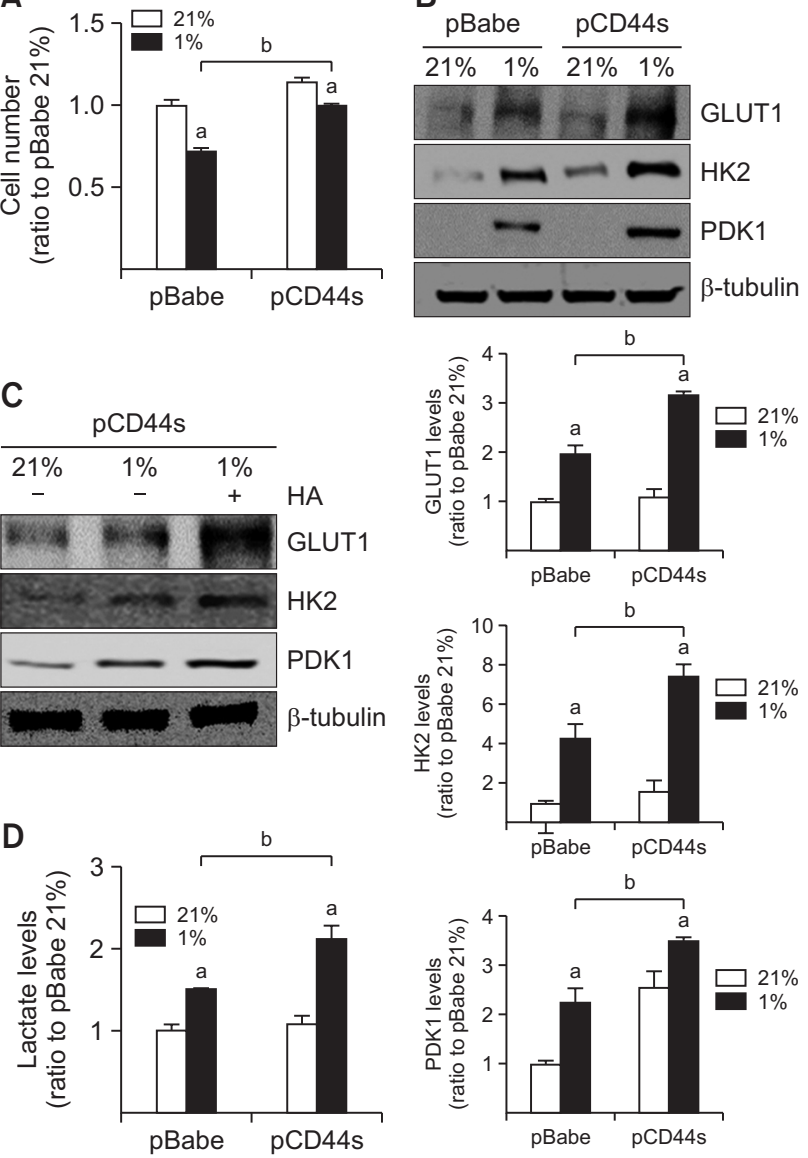

$\mathrm{E}$

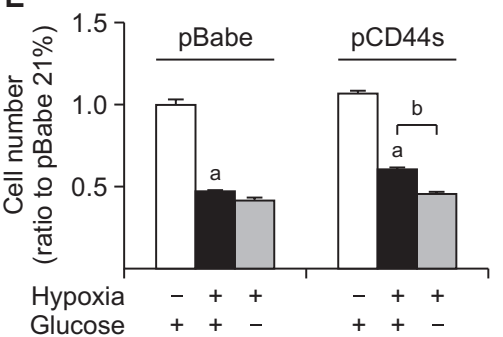

Fig. 5. CD44s overexpression promotes glycolysis pathway and cell survival under hypoxia. (A) The cell number was counted using an automated cell counter after incubation of pCD44s- and pBabeMCF7 cells in normoxic or hypoxic conditions for $24 \mathrm{~h}$. Values are represented as mean \pm SE from three independent experiments. (B) Levels of GLUT1, HK2, and PDK1 were detected after a 24 $\mathrm{h}$ hypoxic incubation. Quantified results are from three independent experiments. Values are represented as mean \pm SE. (C) The pCD44s-MCF7 cells were incubated with HA $(50 \mu \mathrm{g} / \mathrm{mL})$ under hypoxic conditions, and the levels of GLUT1, HK2, and PDK1 were monitored using western blotting. (D) Lactate levels were measured in culture media for normoxic or hypoxic pBabe- and pCD44s-MCF7 cells. Data is represented mean \pm SE from three independent experiments. ${ }^{a} p<0.05$ compared to the normoxic cell line. ${ }^{b} p<0.05$ compared to the hypoxic pBabe-MCF7 control. (E) Viable cell number was monitored after the incubation of cells under glucose-free hypoxic conditions for $24 \mathrm{~h}$. Data is represented as mean \pm SE from three independent experiments. ${ }^{a} p<0.05$ compared to the each normoxic cell line. ${ }^{b} p<0.05$ compared to the each hypoxic cell line in the presence of glucose. 
and the inhibition of HA synthesis led to the suppression of metastasis (Morath et al., 2016; Karousou et al., 2017). In clinical studies, CD44 has been suggested as a valuable biomarker for diagnosis and prognosis of various types of cancers (Yan et al., 2015). A meta-analysis of 2,403 patients with gastric cancer showed a positive association of CD44 expression with incidence of metastasis and reduced patients' overall survival (Chen et al., 2014).

Since CD44 senses environmental changes via ECM to induce aggressive tumor phenotypes, the involvement of CD44 in cellular hypoxic response could be an intriguing issue. In fact, accumulating evidence demonstrates an increase in CD44-mediated cellular response under hypoxic conditions. In MDA-MB231 breast cancer cells, hypoxia-mediated HIF-1 $\alpha$ elevation upregulated CD44 expression, and the co-localization of HRE-driven fluorescence and CD44-positive region was observed in MDA-MB-231 tumors (Krishnamachary et al., 2012). Exposure of gastric cancer cells to hypoxic conditions led to the elevation in CD44 expression (Liang et al., 2017). In the current study, we demonstrated that the overexpression of CD44s in non-aggressive breast cancer MCF-7 cells enhanced hypoxia-inducible HIF-1 $\alpha$ accumulation, and thereby exhibited higher HRE-luciferase activity and VEGF expression during hypoxia compared to the control MCF-7. The direct involvement of CD44s in HIF-1 $\alpha$ elevation was confirmed by the HA-mediated augmentation and CD44-silencing. The CD44smediated HIF-1 $\alpha$ augmentation facilitated hypoxia-induced EMT gene expression and cell motility. In addition, as a metabolic benefit, levels of hypoxia-inducible glycolytic enzymes were higher in CD44s-overexpressing MCF-7 cells, and therefore, the number of viable cells was greater in CD44s-high MCF-7 cells after $24 \mathrm{~h}$ hypoxia than that in the control MCF-7. These results suggest that cancer cells with high CD44s expression can show a better adaptive behavior in response to the hypoxic conditions by enhancing HIF- $1 \alpha$ accumulation. On the other hand, the direct involvement of CD44 in cancer cell metabolism has been demonstrated in a recent study; CD44 increased lactate dehydrogenase expression in breast cancer cells and therefore, the silencing of CD44 switched glycolytic cancer metabolism to oxidative phosphorylation (Nam et al., 2016). This report indicates that, in addition to the facilitated adaptive survival in hypoxic condition, CD44 can change cancer metabolism forward glycolytic condition, which supports the role of CD44 in cancer proliferation and survival.

Under hypoxia, several post-translational changes can modify HIF-1 $\alpha$ stability. In particular, the phosphorylation of Ser641 and Ser643 in HIF-1 $\alpha$ protein, mediated by mitogenactivated kinases (MAPKs), facilitates its heterodimerization with ARNT to transactivate HIF-1 $\alpha$ target genes (Kietzmann et al., 2016). The importance of phosphorylation of HIF-1 $\alpha$ has been demonstrated in a previous study by Suzuki et al (2001). In MCF-7 cells, dephosphorylation of HIF-1 $\alpha$ induced apoptosis by forming a complex with the tumor suppressor p53. MAPK pathways are associated with HIF- $1 \alpha$ regulation via additional molecular mechanisms. ERK signaling increases the transcription of HIF- $1 \alpha$ directly and stimulates HIF- $1 \alpha$ translation via phosphorylation of the ribosomal protein S6K (Semenza, 2012; Masoud and Li, 2015). Consistent with these reports suggesting the potential involvement of MAPK signaling in HIF- $1 \alpha$ activation, we identified that ERK pathway is associated with CD44s-mediated HIF-1 $\alpha$ augmentation under hypoxic conditions. Hypoxia-inducible ERK activation was prominent in CD44s-overexpressing MCF-7 cells, and this activation was further enhanced by HA, indicating the augmentation effect of CD44s on hypoxia-inducible ERK activation. Moreover, the treatment of cells with ERK inhibitors blocked the increase in HIF- $1 \alpha$ and VEGF levels under hypoxia, indicating the role of ERK signaling in CD44s-mediated HIF-1 $\alpha$ activation. The MAPK pathway has been known to be one of signaling target of CD44 (Morath et al., 2016; Chen et al., 2018). In KRASactivated mutant mice, CD44 elevation was correlated with the activation of RAF/MEK/ERK signaling, and knockdown of CD44 inhibited ERK activity in non-small-cell lung cancer (Zhao et al., 2013). Silencing of CD44 in breast cancer cells reduced the expression of $\mathrm{c}-\mathrm{Src}$ via the suppression of SP-1 expression, and subsequently inhibited downstream MAPK signaling (Nam et al., 2015). Furthermore, several types of CD44 variant form were known to interact with growth factor receptors such as epidermal growth factor receptor (EGFR) for the activation of ERK signaling (Karousou et al., 2017). These reports suggest the association of CD44 with MAPK pathway activation; however, molecular event linking CD44 and MAPK seems to be context-dependent. As CD44s overexpression did not activate MAPK signaling in normoxic conditions in our MCF-7 system, CD44s appears to enhance ERK activation only in hypoxic-conditions.

Taken together, our results showed the stimulating effect of CD44s on hypoxia-inducible HIF-1 $\alpha$ accumulation via the involvement of ERK activation, which suggests the reciprocal regulation of CD44 and HIF-1 $\alpha$. These phenomena led to the enhancement of HIF-1 $\alpha$-dependent adaptation system, including glucose metabolism and EMT gene expression, and thus, the cell survival and motility were facilitated in CD44shigh hypoxic breast cancer cells.

\section{CONFLICT OF INTEREST}

The authors declare that they have no conflict of interest.

\section{ACKNOWLEDGMENTS}

This study was financially supported by a grant from the National Research Foundation of Korea (NRF) funded by the Korea government (MSIP; NRF-2015R1A2A1A10054384, NRF-2013M3A9B5075839). This study was also supported by the BK21Plus grant of NRF funded by the Korean government (22A20130012250).

\section{REFERENCES}

Bourguignon, L. Y., Peyrollier, K., Xia, W. and Gilad, E. (2008) Hyaluronan-CD44 interaction activates stem cell marker Nanog, Stat3-mediated MDR1 gene expression, and ankyrin-regulated multidrug efflux in breast and ovarian tumor cells. J. Biol. Chem. 283, 17635-17651.

Chen, C., Zhao, S., Karnad, A. and Freeman, J. W. (2018) The biology and role of CD44 in cancer progression: therapeutic implications. J. Hematol. Oncol. 11, 64.

Chen, Y., Fu, Z., Xu, S., Xu, Y. and Xu, P. (2014) The prognostic value of CD44 expression in gastric cancer: a meta-analysis. Biomed. Pharmacother. 68, 693-697.

Cho, S. H., Park, Y. S., Kim, H. J., Kim, C. H., Lim, S. W., Huh, J. W., 
Lee, J. H. and Kim, H. R. (2012) CD44 enhances the epithelialmesenchymal transition in association with colon cancer invasion. Int. J. Oncol. 41, 211-218.

Guillemin, K. and Krasnow, M. A. (1997) The hypoxic response: huffing and HIFing. Cell 89, 9-12.

Huh, J. W., Kim, H. R., Kim, Y. J., Lee, J. H., Park, Y. S., Cho, S. H. and Joo, J. K. (2009) Expression of standard CD44 in human colorectal carcinoma: association with prognosis. Pathol. Int. 59, 241-246.

Kang, M. H., Choi, S. and Kim, B. H. (2017) Skin wound healing effects and action mechanism of acai berry water extracts. Toxicol. Res. 33, 149-156.

Karousou, E., Misra, S., Ghatak, S., Dobra, K., Gotte, M., Vigetti, D., Passi, A., Karamanos, N. K. and Skandalis, S. S. (2017) Roles and targeting of the HAS/hyaluronan/CD44 molecular system in cancer. Matrix Biol. 59, 3-22.

Kietzmann, T., Mennerich, D. and Dimova, E. Y. (2016) Hypoxia-inducible factors (HIFs) and phosphorylation: impact on stability, localization, and transactivity. Front. Cell Dev. Biol. 4, 11.

Kim, T. H., Hur, E. G., Kang, S. J., Kim, J. A., Thapa, D., Lee, Y. M., Ku, S. K., Jung, Y. and Kwak, M. K. (2011) NRF2 blockade suppresses colon tumor angiogenesis by inhibiting hypoxia-induced activation of HIF-1alpha. Cancer Res. 71, 2260-2275.

Krishnamachary, B., Penet, M. F., Nimmagadda, S., Mironchik, Y., Raman, V., Solaiyappan, M., Semenza, G. L., Pomper, M. G. and Bhujwalla, Z. M. (2012) Hypoxia regulates CD44 and its variant isoforms through HIF-1alpha in triple negative breast cancer. PLoS ONE 7, e44078.

Liang, D., Ma, Y., Liu, J., Trope, C. G., Holm, R., Nesland, J. M. and Suo, Z. (2012) The hypoxic microenvironment upgrades stem-like properties of ovarian cancer cells. BMC Cancer 12, 201.

Liang, G., Li, S., Du, W., Ke, Q., Cai, J. and Yang, J. (2017) Hypoxia regulates CD44 expression via hypoxia-inducible factor-1alpha in human gastric cancer cells. Oncol. Lett. 13, 967-972.

Louie, E., Nik, S., Chen, J. S., Schmidt, M., Song, B., Pacson, C., Chen, X. F., Park, S., Ju, J. and Chen, E. I. (2010) Identification of a stem-like cell population by exposing metastatic breast cancer cell lines to repetitive cycles of hypoxia and reoxygenation. Breast Cancer Res. 12, R94.

Masoud, G. N. and Li, W. (2015) HIF-1alpha pathway: role, regulation and intervention for cancer therapy. Acta Pharm. Sin. B 5, 378-389.

Mima, K., Okabe, H., Ishimoto, T., Hayashi, H., Nakagawa, S., Kuroki, H., Watanabe, M., Beppu, T., Tamada, M. and Nagano, O. (2012) CD44s Regulates the TGF- $\beta$-mediated mesenchymal phenotype and is associated with poor prognosis in patients with hepatocellular carcinoma. Cancer Res. 72, 3414-3423.

Morath, I., Hartmann, T. N. and Orian-Rousseau, V. (2016) CD44: more than a mere stem cell marker. Int. J. Biochem. Cell Biol. 81, 166-173.

Myszczyszyn, A., Czarnecka, A. M., Matak, D., Szymanski, L., Lian, F., Kornakiewicz, A., Bartnik, E., Kukwa, W., Kieda, C. and Szczylik, C. (2015) The role of hypoxia and cancer stem cells in renal cell carcinoma pathogenesis. Stem Cell Rev. 11, 919-943.

Nam, K., Oh, S., Lee, K. M., Yoo, S. A. and Shin, I. (2015) CD44 regulates cell proliferation, migration, and invasion via modulation of c-Src transcription in human breast cancer cells. Cell. Signal. 27, 1882-1894.

Nam, K., Oh, S. and Shin, I. (2016) Ablation of CD44 induces glycolysis-to-oxidative phosphorylation transition via modulation of the c-
Src-Akt-LKB1-AMPKalpha pathway. Biochem. J. 473, 3013-3030.

Naor, D., Nedvetzki, S., Golan, I., Melnik, L. and Faitelson, Y. (2002) CD44 in cancer. Crit. Rev. Clin. Lab. Sci. 39, 527-579.

Ohashi, R., Takahashi, F., Cui, R., Yoshioka, M., Gu, T., Sasaki, S., Tominaga, S., Nishio, K., Tanabe, K. K. and Takahashi, K. (2007) Interaction between CD44 and hyaluronate induces chemoresistance in non-small cell lung cancer cell. Cancer Lett. 252, 225-234.

Park, J. H., Choi, B. H., Ku, S. K., Kim, D. H., Jung, K. A., Oh, E. and Kwak, M. K. (2017) Amelioration of high fat diet-induced nephropathy by cilostazol and rosuvastatin. Arch. Pharm. Res. 40, 391-402.

Patrawala, L., Calhoun, T., Schneider-Broussard, R., Li, H., Bhatia, B., Tang, S., Reilly, J., Chandra, D., Zhou, J. and Claypool, K. (2006) Highly purified CD44+ prostate cancer cells from xenograft human tumors are enriched in tumorigenic and metastatic progenitor cells. Oncogene 25, 1696-1708.

Ryoo, I. G., Choi, B. H., Ku, S. K. and Kwak, M. K. (2018) High CD44 expression mediates p62-associated NFE2L2/NRF2 activation in breast cancer stem cell-like cells: Implications for cancer stem cell resistance. Redox Biol. 17, 246-258.

Semenza, G. L. (1999) Regulation of mammalian O2 homeostasis by hypoxia-inducible factor 1. Annu. Rev. Cell Dev. Biol. 15, 551-578.

Semenza, G. L. (2004) Intratumoral hypoxia, radiation resistance, and HIF-1. Cancer Cell 5, 405-406.

Semenza, G. L. (2012) Hypoxia-inducible factors: mediators of cancer progression and targets for cancer therapy. Trends Pharmacol. Sci. $33,207-214$.

Semenza, G. L. (2013) HIF-1 mediates metabolic responses to intratumoral hypoxia and oncogenic mutations. J. Clin. Invest. 123, 3664-3671.

Shang, Z., Cai, Q., Zhang, M., Zhu, S., Ma, Y., Sun, L., Jiang, N., Tian, J., Niu, X., Chen, J., Sun, Y. and Niu, Y. (2015) A switch from $\mathrm{CD} 44(+)$ cell to EMT cell drives the metastasis of prostate cancer. Oncotarget 6, 1202-1216.

Suzuki, H., Tomida, A. and Tsuruo, T. (2001) Dephosphorylated hypoxia-inducible factor 1 alpha as a mediator of p53-dependent apoptosis during hypoxia. Oncogene 20, 5779-5788.

Tsukita, S., Oishi, K., Sato, N., Sagara, J., Kawai, A. and Tsukita, S. (1994) ERM family members as molecular linkers between the cell surface glycoprotein CD44 and actin-based cytoskeletons. J. Cell Biol. 126, 391-401.

Weidemann, A. and Johnson, R. S. (2008) Biology of HIF-1alpha. Cell Death Differ. 15, 621-627.

Yan, Y., Zuo, X. and Wei, D. (2015) Concise review: emerging role of CD44 in cancer stem cells: a promising biomarker and therapeutic target. Stem Cells Transl. Med. 4, 1033-1043.

Yoon, C., Park, D. J., Schmidt, B., Thomas, N. J., Lee, H.-J., Kim, T. S., Janjigian, Y. Y., Cohen, D. J. and Yoon, S. S. (2014) CD44 expression denotes a subpopulation of gastric cancer cells in which Hedgehog signaling promotes chemotherapy resistance. Clin. Cancer Res. 20, 3974-3988.

Zhang, Z., Filho, M. S. and Nor, J. E. (2012) The biology of head and neck cancer stem cells. Oral Oncol. 48, 1-9.

Zhao, P., Damerow, M. S., Stern, P., Liu, A. H., Sweet-Cordero, A., Siziopikou, K., Neilson, J. R., Sharp, P. A. and Cheng, C. (2013) CD44 promotes Kras-dependent lung adenocarcinoma. Oncogene 32, 5186-5190.

Zöller, M. (2011) CD44: can a cancer-initiating cell profit from an abundantly expressed molecule? Nat. Rev. Cancer 11, 254-267. 